\title{
Laser manipulation of iron for nanofabrication
}

\author{
G. Myszkiewicz, J. Hohlfeld, ${ }^{\text {a) }}$ A. J. Toonen, A. F. Van Etteger, O. I. Shklyarevskii, \\ W. L. Meerts, and Th. Rasing ${ }^{\text {b) }}$ \\ NSRIM, Radboud University Nijmegen, Toernooiveld 1, 6525 ED Nijmegen, the Netherlands \\ E. Jurdik \\ Gasunie Research, Energieweg 17, 9743 AN Groningen, the Netherlands
}

(Received 12 July 2004; accepted 9 September 2004)

\begin{abstract}
We fabricate iron nanolines by depositing an atomic beam of iron through a far-off resonant laser standing wave (SW) onto a glass-ceramic substrate. The laser SW is tuned $200 \mathrm{MHz}$ above the ${ }^{5} D_{4} \rightarrow{ }^{5} F_{5}^{o 56} \mathrm{Fe}$ transition at a vacuum wavelength of $372.099 \mathrm{~nm}$. The resulting nanolines exhibit a period of $186 \mathrm{~nm}$, a height above the background of $8 \mathrm{~nm}$ and a full width at half maximum of $95 \mathrm{~nm}$. These nanostructures cover a surface area of $\simeq 1.6 \times 0.4 \mathrm{~mm}^{2}$, corresponding to $\simeq 8600$ iron lines with a length of $\simeq 400 \mu \mathrm{m}$. (C) 2004 American Institute of Physics. [DOI: 10.1063/1.1811804]
\end{abstract}

One of the most important topics of modern magnetism concerns the fabrication of periodic magnetic nanostructures. These structures constitute model systems for the study of magnetic interactions and switching behavior, ${ }^{1}$ show phenomena-like magnetic photonic band gaps ${ }^{2}$ and will be required for future magnetic data storage at ultrahigh areal densities. Despite the fundamental and technological interest, the fabrication of macroscopic, uniform arrays of welldefined, high-resolution magnetic nanostructures remains a challenge. It is in this spirit that nanofabrication via atom optics attracted our interest as it allows for an inherently parallel, ultrahigh vacuum compatible fabrication of nanostructures with unprecedented periodicity. 3,4

Nanofabrication via atom optics is based on the interaction between an atomic beam and a far-off resonant laser standing wave (SW). The atoms, when crossing the laser SW, experience a spatially varying optical potential that, in the first approximation, follows the SW intensity profile. ${ }^{5}$ When the laser frequency is tuned above (below) an atomic resonance, the atoms gather in the regions of low (high) light intensities. A substrate is then placed into the modulated atomic beam and nanostructures with a subwavelength period are grown. However, the structure width deteriorates fast with an increasing divergence of the atomic beam. ${ }^{6}$ Moreover, the SW optical potential is extremely shallow and so the atomic velocities along the SW direction must be low for the atoms to be "trapped" in SW nodes (antinodes). For these two reasons, a high degree of collimation of the atomic beam is required. In practice, laser cooling schemes are applied as they lead to extremely well-collimated atomic beams with no loss of flux. ${ }^{7}$ It is this laser cooling requirement that has limited nanofabrication via atom optics to just a few atomic species with a suitable optical transition. So far, laserfocused nanofabrication was demonstrated only with sodium, ${ }^{8}$ chromium, ${ }^{3}$ aluminum ${ }^{9}$ and ytterbium ${ }^{10}$ in the direct deposition regime, and with cesium ${ }^{11}$ via lithography with a self-assembled monolayer as the resist. We note that all these atoms possess a closed (or almost closed) optical transition that allows for efficient laser collimation.

\footnotetext{
${ }^{a)}$ Present address: Seagate Research, 1251 Waterfront Place, Pittsburgh, Pennsylvania 15222.

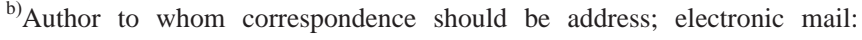
th.rasing@science.ru.nl
}

In this letter we demonstrate atom-optical nanofabrication with iron. An iron atomic beam is deposited onto a glass-ceramic substrate through a one-dimensional laser SW tuned $200 \mathrm{MHz}$ above the ${ }^{5} D_{4} \rightarrow{ }^{5} F_{5}^{o}{ }^{56} \mathrm{Fe}$ transition at a vacuum wavelength of $372.099 \mathrm{~nm}$. The nanoscopically corrugated surface consists of highly uniform iron nanolines with a period of $186 \mathrm{~nm}$, a full width at half maximum (FWHM) of $95 \mathrm{~nm}$ and a height (above the background) of $8 \mathrm{~nm}$. About 8600 iron lines with a length of $\simeq 400 \mu \mathrm{m}$ are grown in a single deposition run of $30 \mathrm{~min}$. We mention here that a different approach to the fabrication of periodic iron nanowires was applied by Tulchinsky et al., ${ }^{12}$ who employed laser-focused chromium nanolines as a shadow mask for an iron evaporator. In contrast, our nonlithographic approach relies on direct deposition of laser-manipulated iron.

For laser manipulation, iron is a considerably more difficult element than any of the atoms used for atom-optical nanofabrication so far. Naturally occurring isotopes of iron are: ${ }^{54} \mathrm{Fe}$ (abundance $\left.5.8 \%\right),{ }^{56} \mathrm{Fe}(91.8 \%),{ }^{57} \mathrm{Fe}(2.1 \%)$ and ${ }^{58} \mathrm{Fe}(0.3 \%)$. The dominant isotope ${ }^{56} \mathrm{Fe}$ has a nuclear spin $I=0$ and thus no hyperfine structure. The most suitable optical transition for laser collimation and SW focusing is the ${ }^{5} D_{4} \rightarrow{ }^{5} F_{5}^{o}$ resonance with a transition wavelength of $\lambda$ $=372.099 \mathrm{~nm}$, a natural linewidth $\Gamma / 2 \pi=2.6 \mathrm{MHz}$ and a saturation intensity $I_{S}=62 \mathrm{~W} / \mathrm{m}^{2}$. However, this is not a closed transition as considerable, dipole-allowed leaks exist from the upper state to the metastable states ${ }^{5} F_{5}$ and ${ }^{5} F_{4}$. Repumping from these two states back into ${ }^{5} F_{5}^{o}$ at 501.35 and $512.88 \mathrm{~nm}$ is an option to enhance the collimation efficiency.

For our experiment, the ultraviolet laser light was obtained by frequency doubling the output of a titanium-doped sapphire laser in an external enchancement doubling cavity based on a lithium triborate (LBO) crystal. This optical system for iron was built by upgrading the optics and replacing the LBO crystal in our earlier chromium setup (425 $\rightarrow 372 \mathrm{~nm}) .{ }^{13} \mathrm{~A}$ thermal beam of iron atoms was produced from a high temperature effusion cell held at $2150 \mathrm{~K}$. At these high temperatures iron becomes extremely corrosive and reactive. A beryllium oxide crucible filled with iron powder was used. The fraction of ${ }^{56} \mathrm{Fe}$ atoms that remain in the ground state ${ }^{5} D_{4}$ at $2150 \mathrm{~K}$ is $46 \%$. The other atoms are thermally excited into the other ${ }^{5} \mathrm{D}$ levels. The vacuum pres- 


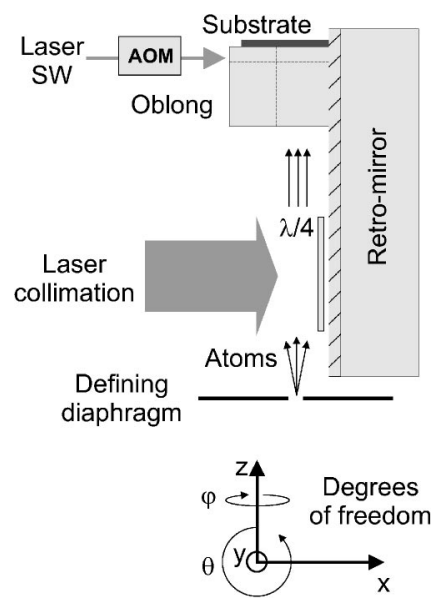

FIG. 1. In vacuo optics including the sample holder. The required degrees of freedom of the unit are also shown.

sure in the course of deposition was below $10^{-6}$ mbar. After the iron beam passed a defining diaphragm of 1.5 $\times 1.5 \mathrm{~mm}^{2}$, it entered the deposition chamber.

In the deposition chamber an in vacuo optical unit is mounted. It is schematically shown in Fig. 1. The required degrees of freedom of the unit are three translations along $x$, $y$ and $z$, and two rotations around $y$ and $z$, respectively. The mirror is a zerodur substrate coated with enhanced aluminum. A glass oblong serves as the sample holder. This oblong was cut from a prism with the right angle specified to within $\pm 0.015 \mathrm{mrad}$. The high quality optical faces of the oblong are used as the reference for the angular adjustment of the sample with respect to the mirror. The sample rests on the top face of the oblong. The atom beam is along $z$ and the laser SW along $x$. Two grooves-one for the atoms and the other one for the laser SW-were cut into the oblong. A quarter-wave $(\lambda / 4)$ plate is located in front of the mirror at the position where laser collimation takes place. Using this $\lambda / 4$ plate a polarization gradient scheme of laser cooling can be created. The optical parts of the in vacuo unit are mounted into a holder made of high-strength aluminum that is in turn mounted onto a vacuum manipulator. This manipulator provides all degrees of freedom required to align the experiment.

The laser is stabilized at a frequency near the ${ }^{5} D_{4}$ $\rightarrow{ }^{5} F_{5}^{o}{ }^{56} \mathrm{Fe}$ transition for which laser collimation is optimized. In our iron experiment, the detuning from the resonance was determined to $(-2 \pm 0.5) \Gamma$. The frequency stabilization scheme is based on the detection of a laser-induced fluorescence (LIF) signal from the iron beam crossing a probe laser. The fluorescence from the intersection is then imaged onto a split photodiode that provides a dispersion signal for the locking electronics. ${ }^{6}$ The collimation angle of the laser-cooled iron beam was optimized empirically. We found out that the best results were obtained with no polarization gradients and no magnetic fields applied. The laser beam used for cooling contained a power of $24 \mathrm{~mW}$ and its $1 / e^{2}$ intensity full widths were $20 \mathrm{~mm}$ along $z$ and $3 \mathrm{~mm}$ along $y$. The fraction of the atomic beam we were able to detect by LIF after cooling exhibited a FWHM collimation angle $\alpha=0.95 \pm 0.10 \mathrm{mrad}$. We note here that these "detectable" atoms are those ones that then couple to the SW laser field. No repumping scheme back to ${ }^{5} F_{5}^{o}$ was applied. field. No repumping scheme back to ${ }^{o} F_{5}$ was applied. Surface growth effects. In addition to iron-on-substrate and
Downloaded 10 Jun 2008 to 131.174 .40 .109 . Redistribution subject to AlP license or copyright; see http://apl.aip.org/apl/copyright.jsp

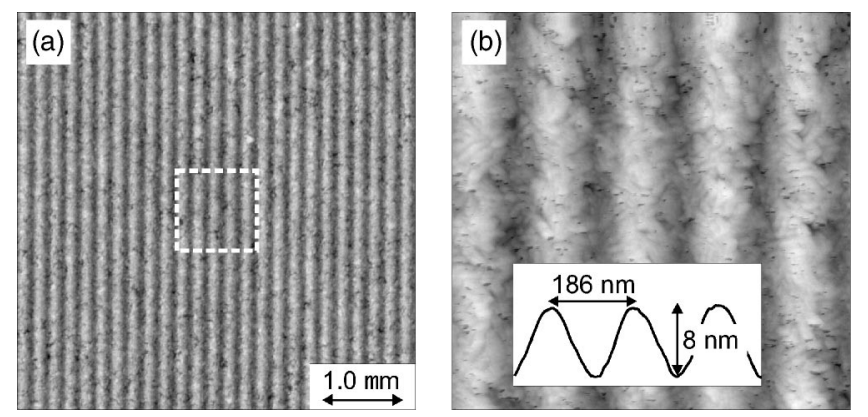

FIG. 2. Laser-focused nanolines of iron. AFM scans: (a) $5 \times 5 \mu \mathrm{m}^{2}$, (b) 1 $\times 1 \mu \mathrm{m}^{2}$. (b) Corresponds to the surface area encapsulated in the white square in (a). Inset in (b): Structure profile averaged over $1 \mu \mathrm{m}$ along the lines.

The SW laser beam was tuned $\simeq 200 \mathrm{MHz}$ above the involved iron resonance by an acousto-optic modulator. The SW contained a laser power of $84 \mathrm{~mW}$ and was focused down to $1 / e^{2}$ intensity full widths of $\simeq 200 \mu \mathrm{m}$ along $y$ and $\simeq 80 \mu \mathrm{m}$ along $z$. At this SW optical intensity, the effect of the SW on the atoms was clearly visible by LIF imaging of the iron beam following a free propagation distance of $\simeq 700 \mathrm{~mm}$ from the SW. The in vacuo optical unit is moved into the iron beam such that the atoms can pass through the vertical groove in the sample holder (Fig. 1). A glass-ceramic substrate was used with a root-mean-square surface roughness of $<5 \AA$. While the deposition was in progress, the SW was cut by the substrate surface at its center. The deposition time was $30 \mathrm{~min}$.

The fabricated sample was subsequently investigated by means of atomic force microscopy (AFM) ex vacuo. A 5 $\times 5 \mu \mathrm{m}^{2}$ AFM scan taken at the center of the iron patch is shown in Fig. 2(a). A highly uniform array of iron nanolines with a period of $\lambda / 2 \simeq 186 \mathrm{~nm}$ is apparent. In Fig. 2(b) a 1 $\times 1 \mu \mathrm{m}^{2}$ AFM scan of the surface area encapsulated in the white square in Fig. 2(a) is presented. The structure profile averaged over $1 \mu \mathrm{m}$ along the lines is shown in the inset of Fig. 2(b). The average structure FWHM and height (above the background) evaluate to $\simeq 95$ and $8 \mathrm{~nm}$, respectively. Neither the images nor the line profiles were corrected for the AFM tip effects as their contribution to the measured structure broadening is estimated to be negligible, akin to earlier studies of laser-focused chromium nanolines by Anderson et al. ${ }^{6}$ In addition, the thickness of the background layer was measured to be $25 \pm 5 \mathrm{~nm}$ using an ellipsometer. This implies a height-to-background ratio of $\simeq 1: 3$.

At this point it is interesting to compare the fabricated nanostructures with the iron flux distribution expected from theoretical considerations. To this end, we calculated the atomic trajectories in a laser SW. The Newton equation of motion with a force due to the SW optical potential was integrated for $10^{6}$ random iron atoms with initial conditions corresponding to the Maxwell-Boltzmann statistics of a thermal $(2150 \mathrm{~K})$ iron beam that is collimated in the transverse direction to $0.95 \mathrm{mrad}$. The laser SW parameters were also set equal to those of the experiment. We estimate that only about $11 \%$ of atoms from the iron beam are affected by the SW. ${ }^{14}$ The final flux distribution at the substrate surface then exhibits an FWHM of $30 \mathrm{~nm}$ and a height-to-background ratio of 1:3. The discrepancy between the measured and modeled structure widths can most likely be attributed to surface growth effects. ${ }^{6}$ In addition to iron-on-substrate and 
iron-on-iron growth, the presence of different contaminants during the deposition and subsequent investigation of the sample ex vacuo could also have contributed to a considerable structure broadening.

We also performed magnetic measurements on the fabricated sample. However, taking into account the large background layer, no signature of individual iron nanolines was expected. Indeed, both magnetic force microscopy and magneto-optical Kerr measurements revealed a ferromagnetic behavior with in-plane easy axis that was independent from the position on the iron patch (irrespective of the presence of nanolines). The drawback of the background layer could be overcome by nanofabrication of magnetic materials with periodically varying composition and thus magnetic properties. This can be achieved by selective manipulation of iron during simultaneous deposition of a number of other species. At this moment, we are performing a study that will allow us to engineer a material with periodically modulated magnetization that might be of interest for applications in magnetic data storage.

In conclusion, we have demonstrated nanofabrication via atom optics with iron. The iron nanostructures exhibited a period of $186 \mathrm{~nm}$, a height above the background of $8 \mathrm{~nm}$ and a FWHM of $95 \mathrm{~nm}$ as determined with an AFM. The thickness of the background layer was estimated to about $25 \mathrm{~nm}$. Considerably narrower structures with a smaller background are feasible from both atom-optical and surface growth perspectives. Repumping from the metastable states ${ }^{5} F$ back into the upper atomic level ${ }^{5} F_{5}^{o}$ would certainly help to achieve better collimation angles and higher populations in the ground state ${ }^{5} D_{4}$. Then, atom optics would work better and considerably narrower nanostructures could be grown. In addition, experiments carried out in a clean UHV environment would allow for a better control of the surface growth processes with a possibly positive impact on the ultimate abilities of the technique. Probably the most limiting factor of all is the thermal population of the ${ }^{5} D$ states, other than the ground state ${ }^{5} D_{4}$, at oven temperatures that are required for a reasonably fast experiment. In order to solve this problem a considerable investment into the laser infrastructures would be required that can only hardly be justified at the present stage of development of nanofabrication via atom optics.
We note here that, independently from us, the group of Professor K.A.H. van Leeuwen from the Eindhoven University of Technology recently succeeded in fabricating iron nanolines via atom optics. ${ }^{15}$

Part of this work was supported by the Stichting voor Fundamenteel Onderzoek der Materie (FOM) that is financially supported by the Nederlandse Organisatie voor Wetenschappelijk Onderzoek (NWO).

${ }^{1}$ Z. K. Wang, H. M. Kuok, S. C. Nig, D. J. Lockwood, M. G. Cottam, K. Nielsch, R. B. Wehrspohn, and U. Gösele, Phys. Rev. Lett. 89, 027201 (2002).

${ }^{2}$ A. Saib, D. Vanhoenacker-Janvier, I. Huynen, A. Encinas, L. Piraux, E. Ferain, and R. Legras, Appl. Phys. Lett. 83, 2378 (2003).

${ }^{3}$ J. J. McClelland, R. E. Scholten, E. C. Palm, and R. J. Celotta, Science 262, 877 (1993).

${ }^{4}$ J. J. McClelland, W. R. Anderson, C. C. Bradley, M. Walkiewicz, R. J. Celotta, E. Jurdik, and R. D. Deslattes, J. Res. Natl. Inst. Stand. Technol. 108, 99 (2003).

${ }^{5}$ J. P. Gordon and A. Ashkin, Phys. Rev. A 21, 1606 (1980).

${ }^{6}$ W. R. Anderson, C. C. Bradley, J. J. McClelland, and R. J. Celotta, Phys. Rev. A 59, 2476 (1999).

${ }^{7}$ R. E. Scholten, R. Gupta, J. J. McClelland, and R. J. Celotta, Phys. Rev. A 55, 1331 (1997).

${ }^{8}$ G. Timp, R. E. Behringer, D. M. Tennant, J. E. Cunningham, M. Prentiss, and K. K. Berggren, Phys. Rev. Lett. 69, 1636 (1992).

${ }^{9}$ R. W. McGowan, D. M. Giltner, and S. A. Lee, Opt. Lett. 20, 2535 (1995).

${ }^{10}$ R. Ohmukai, S. Urabe, and M. Watanabe, Appl. Phys. B: Lasers Opt. 77, 415 (2003).

${ }^{11}$ F. Lison, H.-J. Adams, D. Haubrich, M. Kreis, S. Nowak, and D. Meschede, Appl. Phys. B: Lasers Opt. 65, 419 (1997).

${ }^{12}$ D. A. Tulchinsky, M. H. Kelley, J. J. McClelland, R. Gupta, and R. J. Celotta, J. Vac. Sci. Technol. A 16, 1817 (1998).

${ }^{13}$ E. Jurdik, J. Hohlfeld, A. F. van Etteger, A. J. Toonen, W. L. Meerts, H. van Kempen, and Th. Rasing, J. Opt. Soc. Am. B 19, 1660 (2002).

${ }^{14}$ About $46 \%$ of ${ }^{56} \mathrm{Fe}$ atoms remain in the ground state ${ }^{5} D_{4}$ at an oven temperature of $2150 \mathrm{~K}$. Since the probability for the atoms to decay to the metastable states ${ }^{5} F$ is 1:243 for every scattered photon, only $\simeq 12 \%$ of ${ }^{56} \mathrm{Fe}$ atoms leave the laser cooling region in the ground state, provided a completely saturated transition [E. te Sligte et al., Microelectron. Eng. $\mathbf{6 7 , 6 8 ,} 664$ (2003)]. In addition, there are $8.2 \%$ of other iron isotopes that do not couple to the SW laser field. This implies that only $\simeq 11 \%$ of all atoms are affected by the SW.

${ }^{15}$ E. te Sligte, B. Smeets, K. M. R. van der Stam, R. W. Herfst, P. van der Straten, H. C. W. Beijerinck, and K. A. H. van Leeuwen, Appl. Phys. Lett. (to be published). 